\title{
Pseudomonas associated with Bursaphelenchus xylophilus, its insect vector and the host tree: A role in pine wilt disease?
}

\author{
Marta Salgueiro Alves ${ }^{1}$ (D) | Anabela Pereira ${ }^{1}$ (D) | Cláudia Vicente $^{2}$ (D) | \\ Manuel Mota ${ }^{2,3}$ (i) | Isabel Henriques ${ }^{4}$ (i)
}

\begin{abstract}
${ }^{1}$ Departamento de Biologia e Centro de Estudos do Ambiente e do Mar (CESAM), Universidade de Aveiro, Aveiro, Portugal

${ }^{2}$ NemaLab/ICAAM-Instituto de Ciências Agrárias e Ambientais Mediterrânicas \& Departamento de Biologia, Universidade de Évora, Núcleo de Mitra, Évora, Portugal

${ }^{3}$ Departamento de Ciências da Vida, Universidade Lusófona de Humanidades e Tecnologias, EPCV, Lisboa, Portugal

${ }^{4}$ CESAM \& Department of Life Sciences, Faculty of Sciences and Technology, University of Coimbra, Coimbra, Portugal
\end{abstract}

\section{Correspondence}

Marta Salgueiro Alves, Departamento de Biologia e Centro de Estudos do Ambiente e do Mar (CESAM), Universidade de Aveiro, Aveiro 3810-193, Portugal.

Email: martasalves@ua.pt

Funding information

Fundação para a Ciência e a Tecnologia, Grant/Award Number: IF/00492/2013, PTDC/ BIA-MIC/3768/2012, SFRH/ BD/92999/2013 and UID/AMB/50017; European Regional Development Fund, Grant/Award Number: FCOMP-01-0124FEDER-028368 and POCI-01-0145FEDER-007638

Editor: Paulo Vieira

\begin{abstract}
In this study, we characterized the diversity of Pseudomonas associated with Bursaphelenchus xylophilus, its insect vector (Monochamus galloprovincialis) and its host (Pinus pinaster), by a culture-independent approach using rpoD clone libraries. Clone libraries of Pseudomonas rpoD were obtained from B. xylophilus, M. galloprovincialis and infected $P$. pinaster. Most M. galloprovincialis and B. xylophilus sequences grouped together in the $P$. fluorescens group. Genes related to xenobiotics degradation and phenylacetate synthesis were present in the genomes of the type strains closely related to sequences retrieved from the nematode libraries. Results demonstrated that the nematode, during its life stages inside the tree, maintains a diverse Pseudomonas community that is closely related to the one associated with the insect vector. These bacteria might contribute to degradation of xenobiotics and tree weakening during the nematode tree infection.
\end{abstract}

\section{KEYWORDS}

Bursaphelenchus xylophilus, Monochamus galloprovincialis, pine wilt disease, Pinus pinaster,

Pseudomonas

\section{1 | INTRODUCTION}

Pine wilt disease (PWD) is a devastating disease for coniferous forests in several countries in Europe and Asia. This disease is caused by the plant parasitic nematode Bursaphelenchus xylophilus. Short-distance spread between trees is mediated by cerambycid of the genus Monochamus. The nematodes are carried in the insect tracheae and 
the nematodes relevant in the PWD mechanism (reviewed by Nascimento, Hasegawa, Mota, \& Vicente, 2014). However, the strategies previously applied were based on sequence analysis of the $16 \mathrm{~S}$ rRNA gene, which does not give accurate intra-genus information (Mulet, Bennasar, Lalucat, \& García-Valdes, 2009).

Therefore, the aim of this study was to unravel the diversity of Pseudomonas associated with PWD (the nematode, infected Pinus pinaster trees and insect vector), using rpoD clone libraries, to better understand the roles of this genus of bacteria in this disease. The gene $r p o D$ is a single copy gene reported to provide superior resolution to assess Pseudomonas intra-genus diversity (Mulet et al., 2009).

\section{MATERIAL AND METHODS}

\subsection{Samples and total bacterial DNA extraction}

Samples were collected from infected $P$. pinaster trees in two PWD affected regions in Portugal: Góis $\left(40^{\circ} 09^{\prime} 07.3^{\prime \prime} \mathrm{N} 8^{\circ} 07^{\prime} 34.1^{\prime \prime} \mathrm{W}\right)$ and Comporta $\left(38^{\circ} 22^{\prime} 48.67^{\prime \prime} \mathrm{N} / 8^{\circ} 47^{\prime} 25.00^{\prime \prime} \mathrm{W}\right)$. From each tree trunk, samples comprised sawdust, wood pieces for nematode extraction and logs for arising adult M. galloprovincialis as described in Alves et al. (2018). Total genomic DNA extraction was performed as described in Alves et al. (2018) on samples of nematodes, insects trachea (one insect trachea per sample) and sawdust ( $0.25 \mathrm{~g}$ per sample). A B. xylophilus molecular screening was performed in all samples as described in Alves et al. (2018), and only the positive ones were used. The DNA of 3 nematode samples, three insect samples and four sawdust samples was pooled for each species.

\subsection{Construction and analysis of the Pseudomonas rpoD clone libraries}

Pseudomonas $r p o D$ amplification was performed using the specific primers PsEG30F/ PsEG790R and cycle conditions described in Mulet et al. (2009). Clone libraries were constructed using the TA Cloning Kit, according to the manufacturer's instructions (Invitrogen) and Escherichia coli NZYStar competent cells (NZYTech). Clones were screened by PCR for the presence of fragments with the expected size. Amplicons were sequenced using GATC Biotech services. Sequences that were $100 \%$ identical were grouped to obtain a set of unique sequences (US). The obtained US are available in NCBI platform under the accession numbers MN379315-MN379435. Similarity searches in the GenBank database were performed using the BLAST tool against type strains after sequence editing. Phylogenetic trees were obtained in MEGA 7.0.

\section{3 | RESULTS AND DISCUSSION}

A total of 248 clones were obtained. BLAST analysis of the rpoD gene fragments [sizes between 704 and 710 pb after editing, corresponding to approximately $35 \%$ of the $r p o D$ gene (around

TAB LE 1 Summary of results obtained for clone libraries constructed for Monochamus galloprovincialis, Bursaphelenchus xylophilus and Pinus pinaster regarding the unique sequences (US) detected

\begin{tabular}{|c|c|c|c|c|}
\hline Closest relative type strains & Similarity $(\%)^{a}$ & \multicolumn{3}{|c|}{ Number of US (clones) ${ }^{b}$} \\
\hline Pseudomonas extremorientalis LMG $19695 \mathrm{~T}$ & $95 / 95-96$ & $34(74)$ & $33(48)$ & 0 \\
\hline Pseudomonas yamanorum LMG $27247 T$ & $94-100$ & 0 & $20(28)$ & 0 \\
\hline Pseudomonas extremautralis DMS 17835T & 100 & 0 & $6(8)$ & 0 \\
\hline Pseudomonas japonica JMC 21532T & 84 & 0 & $1(1)$ & 0 \\
\hline Pseudomonas kuykendallii LMG 26364T & $80-95$ & 0 & 0 & $16(23)$ \\
\hline Pseudomonas abietaniphila ATCC 700689T & $90-100$ & 0 & 0 & $3(3)$ \\
\hline Pseudomonas salomonii LMG $22120 \mathrm{~T}$ & 94 & 0 & $1(1)$ & 0 \\
\hline
\end{tabular}

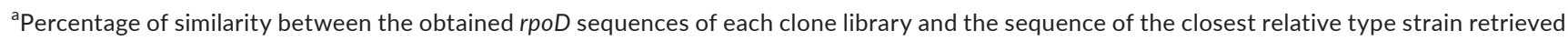
from BLAST.

${ }^{\mathrm{b}}$ Number of US (clones) for each clone library similar to the respective closest type strains.

FIGURE 1 Maximum-likelihood tree illustrating the phylogenetic position of the Pseudomonas rpoD unique sequences (US) obtained in this study, the closest related Pseudomonas type strains and rpoD sequences from Pseudomonas strains from each Pseudomonas group according to Mulet et al. (2012) and Peix et al. (2018). The Cellvibrio japonicus Ueda107 rpoD (CP000934.1) sequence was used as out-group. Bootstrap values of more than 500 (from 1,000 replicates) are indicated at the nodes. The number of US in each compressed subtree is indicated for each species (Bx-Bursaphelenchus xylophilus; $\mathrm{Mg}-$ Monochamus galloprovincialis; $\mathrm{Pp}-$ Pinus pinaster) 


$$
45
$$

Bx: 2 US

74 - Pseudomonas simiae CCUG 50988T

- Pseudomonas lurida P 513/18T

- Pseudomonas extremorientalis LMG 19695T

54 Pseudomonas azotoformans IAM 1603

100 Bx: 6 US

Pseudomonas extremaustralis DSM 17835T

Pseudomonas salomonii LMG 22120T

99 Pseudomonas veronii LMG $17761 \mathrm{~T}$

Pseudomonas fluorescens DSM 50108

Pseudomonas grimontii CIP 106645T

Bx: 6 US

$8699 \mathrm{Bx}: 2$ US

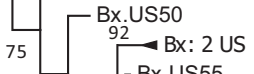

74

Bx.US55

99 1 Bx.US3

86 Pseudomonas yamanorum LMG 27247T

75 - Pseudomonas brenneri DSM 15294T 99 Pseudomonas gessardii CIP 105469T Bx.US63

Bx.US56

Bx.US11

65 Bx.US5

Bx.US59

Bx.US39

Bx.US30

Bx.US52

-Bx.US36

Bx.US46

Bx.US41

Bx.US4

Bx.US32

Pseudomonas fragi ATCC 4973T

Pseudomonas mandelii LMG $21607 T$

8

Pseudomonas koreensis LMG 21318T

69 Pseudomonas jessenii CIP 10574T

Pseudomonas corrugata LMG1276

Pseudomonas chlororaphis PCL1606

Pseudomonas asplenii NCPPB 1947

Pseudomonas agarici NCPPB 1999

99

97

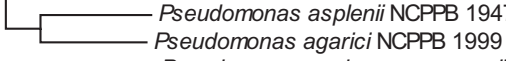

Pseudomonas syringae pv. maculicola

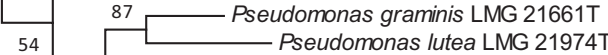

80 Pseudomonas abietaniphila ATCC 700689T

98 Pseudomonas rhizosphaerae LMG 21640T

Pseudomonas rhizosphaerae LMG $21640 \mathrm{~T}$
Pseudomonas japonica JCM 21532T

55

98 Bx.US38

Pseudomonas alkylphenolica KL28

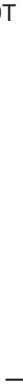

Lineage: $P$. fluorescens

Group: P. fluorescens

Sub-group: $P$. gessardii

Lineage: $P$. fluorescens Group: P. lutea

Lineage: P. fluorescens

Group: P. putida
Lineage: $P$ fluorescens Group: P. fluorescens Sub-group: $P$. fluorescens

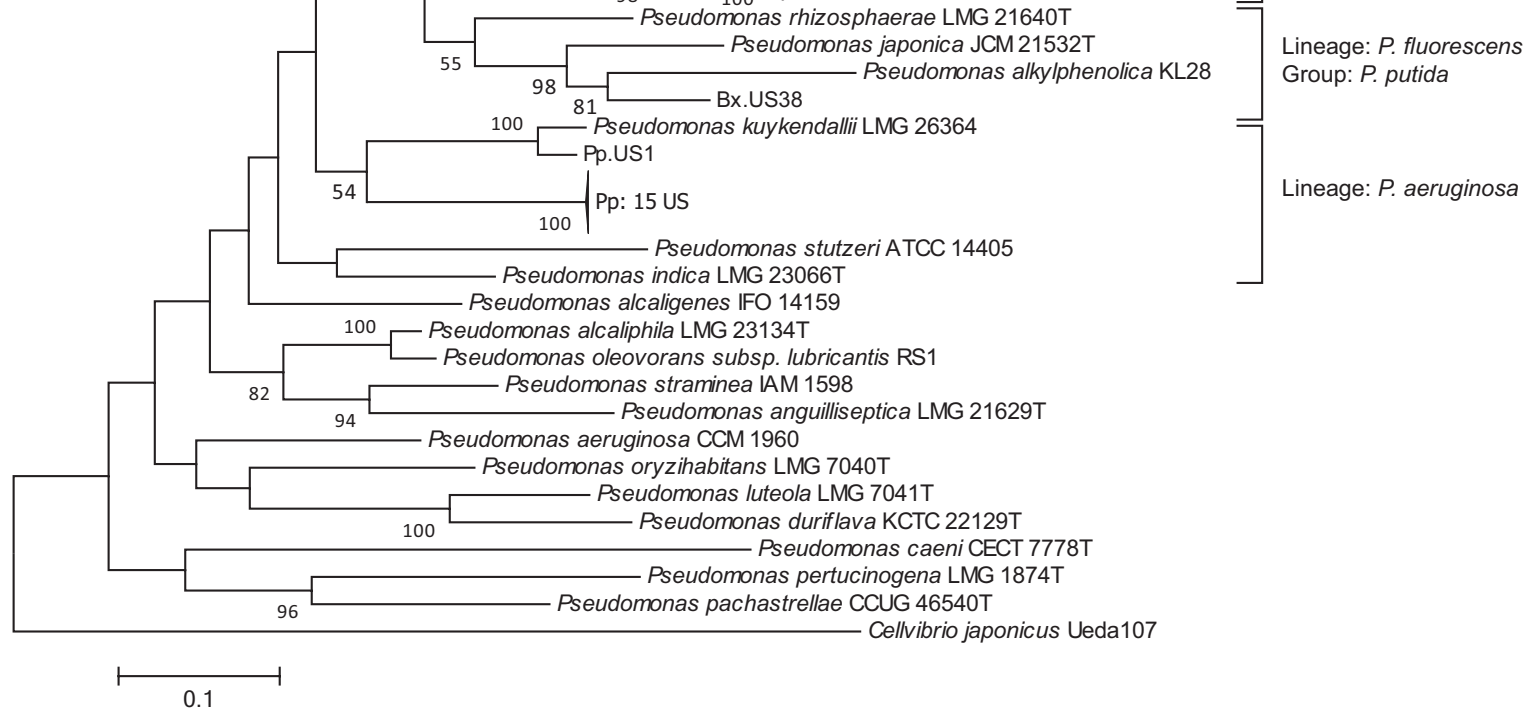

Lineage: $P$. aeruginos

Pseudomonas stutzeri ATCC 14405 
$1850 \mathrm{pb})]$ revealed 202 clones with similarities ranging from $84 \%$ to $100 \%$ to Pseudomonas spp. type strains, 44 clones with simi-

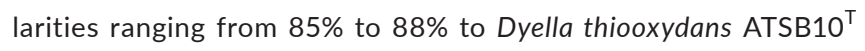
and two clones with $99 \%$ similarities to Serratia ficaria NCTC $12148^{\top}$. The 46 clones that affiliated with Dyella and Serratia belonged to the $P$. pinaster clone library. Sequences affiliating with other bacterial genera (e.g., Alcalinovorax) were occasionally amplified with these primers in previous studies (Mulet et al., 2009). After discarding sequences that did not affiliate with Pseudomonas, 26 clones remained for $P$. pinaster corresponding to 19 US (named Pp.US1-19), 100 for B. xylophilus that corresponded to 68 US (named Bx.US1-68) and 74 clones for M. galloprovincialis (named Mg.US1-74) that corresponded to 34 US. The BLAST analysis against Pseudomonas type strains is listed in Table 1. The phylogenetic tree (Figure 1) was constructed based on the maximum-likelihood method using the rpoD US from the clone libraries, the closest related type strains listed in Table 1 and strains representative of each group and subgroup of the Pseudomonas genus according to Mulet et al. (2012) and Peix, Ramírez-Bahena, and Velázquez (2018). Affiliation of the isolates was based on wellsupported monospecific clades (bootstrap values above 50 ) in the phylogenetic tree obtained. Most of the retrieved sequences for B. xylophilus affiliated with the Pseudomonas fluorescens group (Mulet et al., 2012; Peix et al., 2018) together with all the M. galloprovincialis US. Moreover, the sequences Mg.US8 (comprising 33 clones) and Bx.US7 (three clones) were 100\% identical. P. pinaster US affiliated with the $P$. lutea group (three US) and $P$. aeruginosa lineage (16 US), thus representing a different Pseudomonas community from that observed for the nematode and the insect vector.

Although B. xylophilus was collected from the tree and not from the insect, its associated Pseudomonas community was closely related to the one associated with the insect vector.

Bursaphelenchus xylophilus sequences affiliated with P. alkylphenolica as well as with $P$. yamanorum and $P$. extremaustralis. In the genomes of $P$. yamanorum (accession number LT629793) and P. extremaustralis strains (accession numbers FUYI00000000.1, LT629689 and AHIP00000000.1), we found genes coding for enzymes for the complete degradation of benzoate, a common intermediate in the anaerobic metabolism of toxic compounds, like phenols and other aromatic metabolites produced by the tree as a defence mechanism. Moreover, genes coding for enzymes that could help in nematode protection against oxidative stress were also detected in P. yamanorum and P. extremaustralis genomes (e.g., genes coding for catalases, peroxidases, redox proteins, glutathione S-transferase). The genomes of the four Pseudomonas strains had genes coding for the necessary enzymes for phenylacetate production. This compound was previously described as being produced by nematode-associated bacteria causing wilting symptoms (Proença, Grass, \& Morais, 2016).

This is the first study analysing the diversity of Pseudomonas associated with PWD. The shared phylotypes between the nematode and the insect vector support the hypothesis that the nematode inherits part of its microbiome from the insect vector keeping it in its life stage inside the tree. The Pseudomonas strains detected may help the nematode in the degradation of xenobiotic compounds found inside the tree host and in tree weakening.

\section{ACKNOWLEDGEMENTS}

Acknowledgements are due to FEDER for funding this research through COMPETE 2020 and to FCT for funding the research project Micronema (PTDC/ BIA-MIC/3768/2012; FCOMP-01-0124FEDER-028368). Thanks are due for the financial support provided to CESAM (UID/AMB/50017 - POCI-01-0145-FEDER-007638), by FCT/MCTES through national funds (PIDDAC), and the co-funding by FEDER, within the PT2020 Partnership Agreement and Compete 2020. FCT also financed Isabel Henriques (FCT Investigator Programme-IF/00492/2013] and Marta Alves [PhD grant SFRH/ BD/92999/2013]. The authors wish to acknowledge Dr. Anthony Moreira (University of Aveiro, Portugal) for the English revision of the manuscript.

\section{ORCID}

Marta Salgueiro Alves (iD https://orcid.org/0000-0003-1060-058X

Anabela Pereira (iD https://orcid.org/0000-0003-2351-1084

Cláudia Vicente (D) https://orcid.org/0000-0002-3865-5358

Manuel Mota iD https://orcid.org/0000-0002-4145-5031

Isabel Henriques iD https://orcid.org/0000-0001-7717-4939

\section{REFERENCES}

Alves, M., Pereira, A., Vicente, C., Matos, P., Henriques, J., Lopes, H., ... Henriques, I. (2018). The role of bacteria in pine wilt disease: Insights from microbiome analysis. FEMS Microbiology Ecology, 94. https://doi. org/10.1093/femsec/fiy077

Mulet, M., Bennasar, A., Lalucat, J., \& García-Valdes, E. (2009). An rpoD based PCR procedure for the identification of Pseudomonas species and for their detection in environmental samples. Molecular and Cellular Probes, 23, 140-147. https://doi.org/10.1016/j. mcp.2009.02.001

Mulet, M., Gomila, M., Scottaa, C., Sánchez, D., Lalucat, J., \& GarcíaValdés, E. (2012). Concordance between whole-cell matrix-assisted laser-desorption/ionization time-of-flight mass spectrometry and multilocus sequence analysis approaches in species discrimination within the genus Pseudomonas. Systematic and Applied Microbiology, 65, 455-464. https://doi.org/10.1016/j.syapm.2012.08.007

Nascimento, F. X., Hasegawa, K., Mota, M., \& Vicente, C. S. L. (2014). Bacterial role in pine wilt disease development - review and future perspectives. Environmental Microbiolology Reports, 7, 51-63.

Peix, A., Ramírez-Bahena, M.-H., \& Velázquez, E. (2018). The current status on the taxonomy of Pseudomonas revisited: An update. Infection Genetics and Evolution, 57, 106-116. https://doi.org/10.1016/j. meegid.2017.10.026 
Proença, D. N., Grass, G., \& Morais, P. V. (2016). Understanding pine wilt disease: Roles of the pine endophytic bacteria and of the bacteria carried by the disease-causing pinewood nematode. MicrobiologyOpen, 6, e415.

Vicente, C., Espada, M., Vieira, P., \& Mota, M. (2012). Pine wilt disease: A threat to European forestry. European Journal of Plant Pathology, 133, 89-99. https://doi.org/10.1007/s10658-011-9924-x
How to cite this article: AlvesMS, PereiraA, VicenteC,

MotaM, Henriquesl. Pseudomonas associated with

Bursaphelenchus xylophilus, its insect vector and the host tree: A role in pine wilt disease?For Path. 2019;00:e12564. https://doi.org/10.1111/efp.12564 